\title{
Synchronous Resection of Parotid Metastasis With Esophagectomy: A Case Report
}

\author{
HIDENORI SUZUKI ${ }^{1}$, TETSUYA ABE ${ }^{2}$, EIICHI SASAKI ${ }^{3}$, TAKUYA NAGAO ${ }^{2}$, \\ AKIHIRO MURASHIMA ${ }^{1}$, HIRONORI FUJIEDA ${ }^{2}$, EIJI HIGAKI $^{2}$ and NOBUHIRO HANAI ${ }^{1}$ \\ ${ }^{1}$ Department of Head and Neck Surgery, Aichi Cancer Center Hospital, Nagoya, Japan; \\ ${ }^{2}$ Department of Gastroenterological Surgery, Aichi Cancer Center Hospital, Nagoya, Japan; \\ ${ }^{3}$ Department of Pathology and Molecular Diagnostics, Aichi Cancer Center Hospital, Nagoya, Japan
}

\begin{abstract}
Background: Preoperative diagnosis of parotid tumor is mainly performed via imaging and cytology to avoid both facial nerve injury and tumor seeding. Synchronous resection of solitary parotid metastasis with esophagectomy for esophageal squamous cell carcinoma is rarely performed. Case Report: This is a case report of a 58-year-old male patient with a left preauricular tumor, which was cytologically diagnosed as squamous cell carcinoma. Esophageal squamous cell carcinoma was pathologically diagnosed following ${ }^{18} \mathrm{~F}$ fluorodeoxyglucose positron emission tomography with computed tomography. His diagnosis was cT2NOMO esophageal squamous cell carcinoma with cT3NOMO parotid carcinoma. Esophagectomy for esophageal cancer, as well as parotidectomy were performed. Pathological diagnosis of pTIbN1M1 (parotid) esophageal squamous cell carcinoma was based on exclusion diagnosis of no primary tumor from several specialists and pathological findings including immunohistochemical staining. Conclusion: Reporting of more cases of rare synchronous metastasectomy and esophagectomy is needed to clarify treatment strategies.
\end{abstract}

Approximately $15-32 \%$ of parotid tumours are carcinomas (1). Parotic cancer is a rare malignant tumor accounting for approximately $0.5 \%$ of all malignancies, with a heterogeneity of $\geq 20$ histologic types (2). Because open biopsy is not recommended for parotid tumors due to the risk of both tumor dissemination and facial nerve palsy, both images and fine-

This article is freely accessible online.

Correspondence to: Hidenori Suzuki, MD, Ph.D., Department of Head and Neck Surgery, Aichi Cancer Center Hospital, Nagoya 464-0021, Japan. Tel: +81 527626111, Fax: +81 527642963, e-mail: hi.suzuki@aichi-cc.jp

Key Words: Esophageal squamous cell carcinoma, parotid metastasis, parotid cancer, synchronous resection, ${ }^{18} \mathrm{~F}$-fluorodeoxyglucose positron emission tomography. needle aspiration cytology as preoperative methods for tumor diagnosis cannot be used for the pathologic diagnosis (2). Parotid carcinoma with extraparenchymal extension such as in soft tissues is classified as clinical T3 according to the tumornode-metastasis (TNM) staging system on the eighth edition of Union for International Cancer Control (UICC $8^{\text {th }}$ ) (3).

Positron emission tomography with computed tomography using ${ }^{18} \mathrm{~F}$-fluorodeoxyglucose $\left({ }^{18} \mathrm{~F}\right.$-FDG-PET/CT) for head and neck imaging are widely used for detecting metastatic disease or synchronous cancer at TNM staging $(4,5)$. Parotid metastasis from esophageal carcinoma is rarer than the lymph nodes, liver, lung, bone, brain, and adrenal glands (6). As distant metastatic disease from esophageal carcinoma is mainly treated by systematic chemotherapy, esophagectomy with synchronous resection is rarely performed for the management of distant metastasis (7). Indeed, only few cases of pathological parotid metastasis from esophageal carcinoma with metachronous parotidectomy following esophagectomy (8) have been reported and only one with biopsy at clinical staging (4). Herein, we report a patient who underwent both synchronous resection of solitary parotid metastasis and esophagectomy.

\section{Case Report}

This case report, conducted in accordance to the Declaration of Helsinki for medical protocols and ethics, was approved by the review board of Aichi Cancer Centre (receipt number: 2020-1-702). A 58-year-old male patient with both a growing tumor and pain at the left preauricular was diagnosed with suspicious malignancy based on the squamous cell carcinoma (SCC) cytology.

Palpation and visual inspection at the first visit to our hospital revealed a preauricular tumor with a maximum size of $32 \mathrm{~mm}$ and neither facial palsy nor skin invasion. Endoscopy did not reveal a primary tumor in the head and neck. Both a dermatologist and an ophthalmologist diagnosed no primary tumor in the skin and eye, respectively. Enhanced 

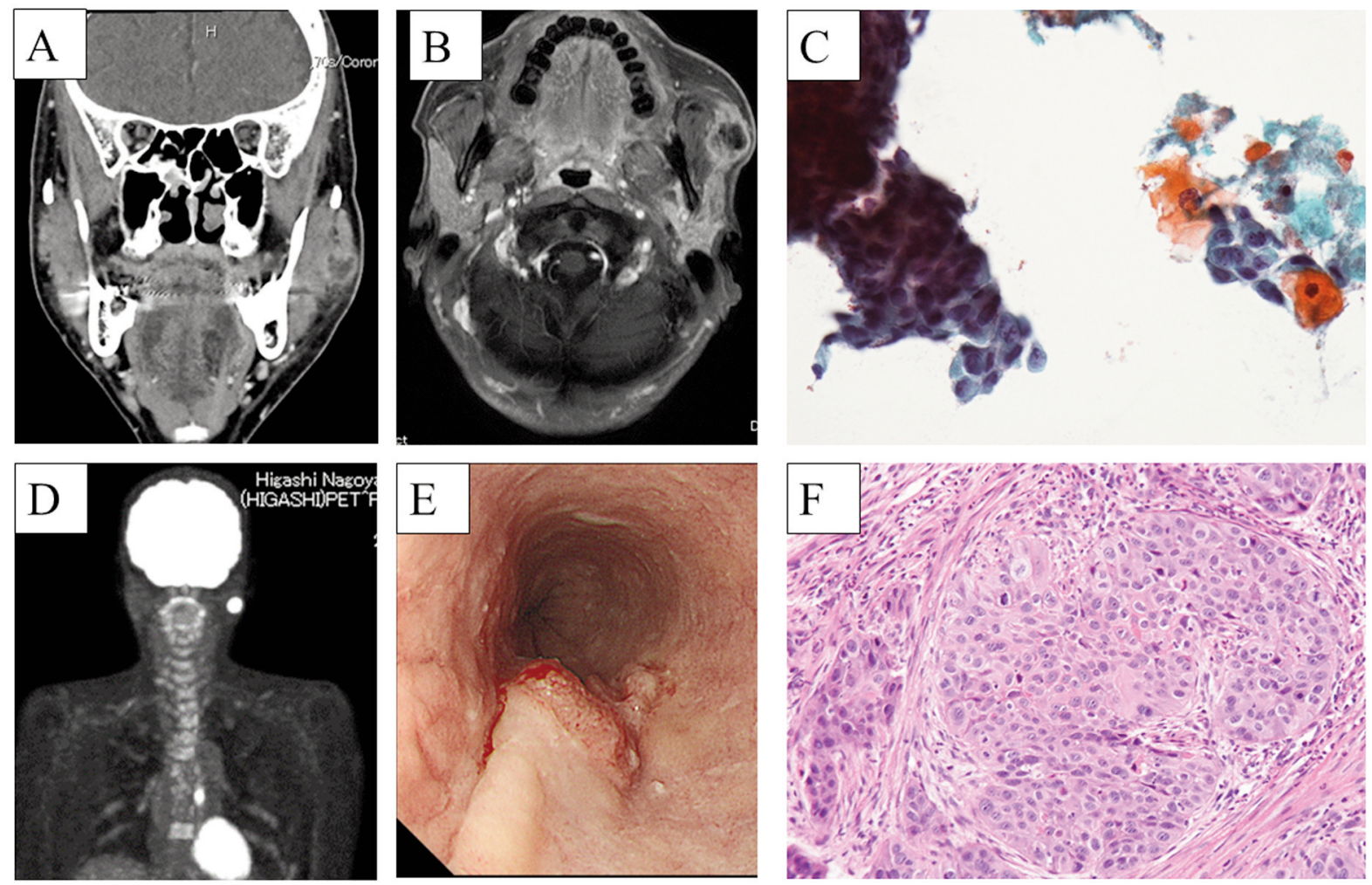

Figure 1. Images before treatment. (A) Enhanced computed tomography, (B) enhanced magnetic resonance imaging, and (C) Papanicolaou staining of the parotid tumor. (D) ${ }^{18}$ F-fluorodeoxyglucose positron emission tomography with computed tomography of parotid and esophageal tumors. (E) endoscopy and $(F)$ Hematoxylin and eosin staining of the esophageal tumor specimen.

computed tomography (CT) and magnetic resonance imaging (MRI) revealed that the left parotid tumor had spread to both subcutaneous tissue and masseter muscle without neck lymphnode metastasis. Whole body ${ }^{18}$ F-FDG-PET/CT revealed abnormal uptake in the parotid tumor, middle thoracic esophagus, lower esophagus, and multiple bones (clavicula, humerus, sternum, rib and spine). Upper digestive endoscopy revealed four esophageal tumors: one lesion with cT2 and three lesions with superficial tumors, and the tumors were biopsied. Enhanced/plane MRI of the cervical-thoracic spine revealed hypercellular marrow based on the absence of tumor recognition. Biopsy specimen of the esophageal tumor was pathologically diagnosed as SCC. Carcinoma cells with squamous differentiation were detected by cytologic analysis of a specimen from the parotid gland tumor. Squamous differentiation is commonly observed in various salivary gland cancers, such as mucoepidermoid carcinoma. Therefore, the diagnosis was cT2N0M0 esophageal SCC with cT3N0M0 parotid carcinoma. Figure 1 shows the pre-treatment images.

The regimen of preoperative chemotherapy was 5fluorouracil of $800 \mathrm{mg} / \mathrm{m}^{2}$ on days $1-5$ and $80 \mathrm{mg} / \mathrm{m}^{2}$ of cisplatin on day 1. After two courses of preoperative chemotherapy, new lesion was not detected in the enhanced/plane whole-body CT. At 97 days following the first visit to our hospital, thoracoscopic esophagectomy with both lymphadenectomy and gastric tube reconstruction for esophageal cancer as well as parotidectomy with dissections of the surrounding tissue, i.e., the skin, subcutaneous fat tissues, masseter muscles and peripheral facial nerve branches, were performed under general anesthesia. At 16 days post-operation, the patient was discharged after resuming oral intake.

Pathologic diagnosis from surgical specimens was esophageal SCC with the maximum size of $12 \mathrm{~mm}$, with two positive lymph-node metastases in the total number of 71 resected lymph nodes. The parotid gland tumor was $27 \mathrm{~mm}$ in size and histologically SCC with extraparenchymal extension (Figure 2A and B). The identical histology between esophageal cancer and parotid gland cancer, taken together with the rarity of pure SCC arising in the parotid gland, suggested that the parotid gland cancer was with a metastasis of esophageal cancer. Additionally, to rule out the possibility of other salivary gland cancers with squamous differentiation or of cervical lymph node metastasis of 

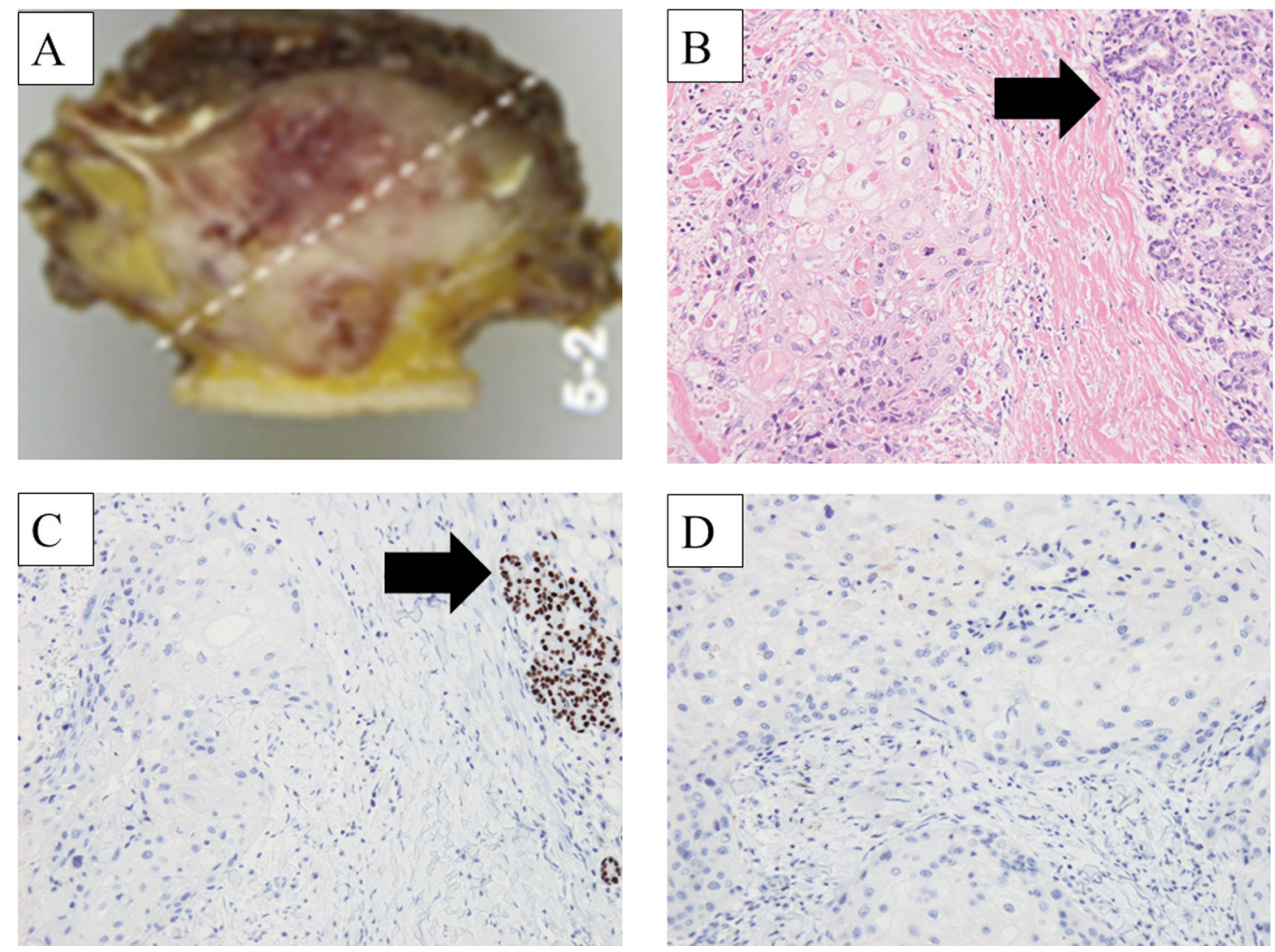

Figure 2. Images from the resected parotid tumor. (A) The surgical specimen. (B) Hematoxylin and eosin staining of the parotid tumor specimen. Negative immunohistochemical staining for $(C)$ SOX10 and (D) p16 of the parotid tumor specimen. Arrows show normal parotid tissues.

unknown primary tumor, immunostaining for SOX10 [BC34, ready-to-use (RTU), Nichirei Biosciences, Tokyo, Japan] and p16 (CINtec Histology Kit, clone E6H4, Ventana, Tucson, AZ, USA) were performed as described in detail elsewhere $(9,10)$. The tumor was negative for SOX10, a representative marker of salivary gland tumor (11), and p16, a surrogate marker of HPV-related cancer (Figure 2C and D). Based on these findings, our patient was evaluated to have pT1bN1M1 (parotid) esophageal SCC. Pathologic surgical margins for esophageal and parotid tumors were negative.

\section{Discussion}

If there is uncertainty regarding the decision of the $\mathrm{T}, \mathrm{N}, \mathrm{M}$ category, the lower of the two possible categories should be selected as the general rule of UICC8th TNM staging (3). Therefore, the parotid malignant tumor in this case was initially diagnosed as a primary parotid cancer, not a metastatic parotid cancer. One stage surgery for parotid cancer has been suggested as the best procedure due to the reduction in tumor seeding by two stage surgery $(1,2)$; therefore, synchronous esophagectomy and parotidectomy for this case was rationally due to the negative pathological surgical margins.

Metastatic parotid carcinomas develop primarily from cutaneous or head and neck carcinomas (12). Parotid metastases from distant sites have been shown to originate from bronchial, renal, and breast cancers among 10,944 salivary gland tumors (13). Moreover, the number of parotid metastasis in a systematic review of 10,049 studies was 2 in 162 esophageal cancer patients with unexpected distant metastasis other than common metastatic sites such as the liver, bone, lung, adrenal gland, and brain (6). Because parotid metastasis from esophageal cancer is rare $(6,12,13)$, this case of parotid metastasis from esophageal SCC was diagnosed based on several findings, i.e., the presence of esophageal SCC, exclusion diagnosis of no primary tumor from several specialists (head and neck, eye and skin) and pathological findings including immunohistochemical staining $(9,10)$. 
Resection of distant metastasis such as from colon cancers have been suggested to improve survival outcomes (14). Metastasectomy including lung metastasectomy in esophageal cancer remains to be reviewed to improve survival outcomes as it is rarely reported (7). Moreover, Depypere LP et al. reported that survival of 10 patients undergoing esophagectomy following preoperative chemotherapy with synchronous resection of unexpected single-organ metastasis (peritoneal: $\mathrm{n}=1$, lung: $\mathrm{n}=5$, pleura: $\mathrm{n}=1$, adrenal: $\mathrm{n}=2$ and pancreas: $\mathrm{n}=1$ ) was longer than the survival of patients with 10 persistent pathologic lymph nodes, and that no patients had undergone both esophagectomy and parotidectomy (15). Therefore, we considered that further case accruement of rare synchronous metastasectomy with esophagectomy is required for appropriate esophageal cancer treatment.

In conclusion, we report a case of pathological parotid metastasis from esophageal cancer based on synchronous resection of parotid malignant tumor with subcutaneous extension and esophagectomy. ${ }^{18}$ F-FDG-PET/CT imaging of the head and neck served to detect a rare case with synchronous resection of a malignant tumor with esophagectomy for esophageal cancer.

\section{Conflicts of Interest}

The Authors have no conflicts of interest to declare in relation to this study.

\section{Authors' Contributions}

HS: was the main operator of the parotid tumor resection, and prepared the manuscript and igures. TA, TN: were the main operators of esophagectomy and revised the manuscript critically, HF, EH: assisted in esophagectomy and revised the manuscript critically. ES: acquired the data. $\mathrm{NH}$ : contributed to the writing of the manuscript.

\section{Acknowledgements}

This study was not supported by a private or a public agency.

\section{References}

1 Gatta G, Guzzo M, Locati LD, McGurk M and Prott FJ: Major and minor salivary gland tumours. Crit Rev Oncol Hematol 152: 102959, 2020. PMID: 32485526. DOI: 10.1016/j.critrevonc. 2020.102959

2 Ettl T, Schwarz-Furlan S, Gosau M and Reichert TE: Salivary gland carcinomas. Oral Maxillofac Surg 16(3): 267-283, 2012. PMID: 22842859. DOI: 10.1007/s10006-012-0350-9

3 Brierley J, Gospodarowicz MD and Wittekind C: TNM classification of malignant tumors. International union against cancer. Oxford, England, Wiley-Blackwell 8th Edition, 2016.
4 Hsieh TC, Wu YC, Sun SS, Yang CF and Kao CH: Parotid gland metastasis from squamous cell carcinoma of esophagus detected by FDG PET/CT. Clin Nucl Med 37(10): 1001-1002, 2012. PMID: 22955076. DOI: 10.1097/RLU.0b013e318263925e

5 Suzuki H, Hasegawa Y, Terada A, Ogawa T, Hyodo I, Suzuki M, Nakashima T, Tamaki T and Nishio M: Limitations of FDG-PET and FDG-PET with computed tomography for detecting synchronous cancer in pharyngeal cancer. Arch Otolaryngol Head Neck Surg 134(11): 1191-1195, 2008. PMID: 19015450. DOI: 10.1001/archotol.134.11.1191

6 Shaheen O, Ghibour A and Alsaid B: Esophageal cancer metastases to unexpected sites: a systematic review. Gastroenterol Res Pract 2017: 1657310, 2017. PMID: 28659974. DOI: $10.1155 / 2017 / 1657310$

7 Jamel S, Tukanova K and Markar S: Detection and management of oligometastatic disease in oesophageal cancer and identification of prognostic factors: A systematic review. World J Gastrointest Oncol 11(9): 741-749, 2019. PMID: 31558978. DOI: $10.4251 /$ wjgo.v11.i9.741

8 Schoneveld JM, Hesp WL and Teune TM: Parotid metastasis from a gastroesophageal carcinoma: report of a case. Dig Surg 24(1): 68-69, 2007. PMID: 17369685. DOI: 10.1159/000100922

9 Suzuki H, Sasaki E, Nishikawa D, Nishikawa D, Koide Y, Beppu $\mathrm{S}$, Terada $\mathrm{H}$ and Hanai N: p16-positive oropharyngeal cancer with rare thyroid metastasis: A case report. J Med Invest 67(1.2): 189191, 2020. PMID: 32378605. DOI: 10.2152/jmi.67.189

10 Sasaki E, Masago K, Fujita S, Suzuki H, Hanai N and Hosoda W: Salivary secretory carcinoma harboring a novel ALK fusion: expanding the molecular characterization of carcinomas beyond the ETV6 gene. Am J Surg Pathol 44(7): 962-969, 2020. PMID: 32205481. DOI: 10.1097/PAS.0000000000001471

11 Hsieh MS, Lee YH and Chang YL: SOX10-positive salivary gland tumors: a growing list, including mammary analogue secretory carcinoma of the salivary gland, sialoblastoma, low-grade salivary duct carcinoma, basal cell adenoma/adenocarcinoma, and a subgroup of mucoepidermoid carcinoma. Hum Pathol 56: 134-142, 2016. PMID: 27327192. DOI: 10.1016/j.humpath.2016.05.021

12 Franzen AM, Günzel T and Lieder A: Parotid gland metastases of distant primary tumours: A diagnostic challenge. Auris Nasus Larynx 43(2): 187-191, 2016. PMID: 26526643. DOI: 10.1016/j.anl.2015.09.010

13 Seifert G, Hennings K and Caselitz J: Metastatic tumors to the parotid and submandibular glands - analysis and differential diagnosis of 108 cases. Pathol Res Pract 181(6): 684-692, 1986. PMID: 3562340. DOI: 10.1016/S0344-0338(86)80044-9

14 Brown KGM and Koh CE: Surgical management of recurrent colon cancer. J Gastrointest Oncol 11(3): 513-525, 2020. PMID: 32655930. DOI: 10.21037 /jgo-2019-ccm-09

15 Depypere LP, Moons J, Lerut TE, Coosemans W, Van Veer H and Nafteux PR: Palliative esophagectomy in unexpected metastatic disease: sense or nonsense? Asian Cardiovasc Thorac Ann 26(7): 552-557, 2018. PMID: 30185072. DOI: 10.1177/ 0218492318797228

Received April 11, 2021

Revised May 5, 2021

Accepted May 7, 2021 